\title{
Pemphigus Vulgaris After COVID-19: a Case of Induced Autoimmunity
}

\author{
Vanessa Lucilia Silveira De Medeiros ${ }^{1,2,3}$ - Antônio Urbano Monteiro-Neto ${ }^{3} \cdot$ David Duarte Timponi França $^{3}$. \\ Rebecca Castelo Branco ${ }^{1}$. Érika Oliveira de Miranda Coelho ${ }^{2,4} \cdot$ Daniela Mayumi Takano $^{5}$
}

Accepted: 18 May 2021 / Published online: 27 May 2021

(C) The Author(s), under exclusive licence to Springer Nature Switzerland AG 2021

\begin{abstract}
Bullae autoimmune diseases can be induced by environmental factors in a genetically susceptible individual, and viruses may be important triggers for this process. Coronavirus disease 2019 (COVID-19) is a multisystemic disease known for developing many types of skin lesions. However, little is known about post-COVID-19 manifestations. A previous healthy male patient, 43 years old, with resistant mediastinal Hodgkin's lymphoma stage II diagnosed in 2019, without treatment at the moment, developed monomorphic flaccid bullae on the trunk 40 days after testing positive for severe acute respiratory syndrome coronavirus 2 (SARS-CoV-2). Although he had risk factors for IgA and paraneoplastic pemphigus, there were no elements for these diseases. The investigation confirmed a pemphigus vulgaris (PV). PV had a good response to treatment with prednisolone 1 $\mathrm{mg} / \mathrm{kg}$ per day, and methotrexate $15 \mathrm{mg}$ subcutaneously was added per week. The patient was discharged with oral methotrexate and prednisolone at the same dose. Prednisolone was decreased by $20 \mathrm{mg}$ per day during the follow-up. Viruses such as herpesvirus, cytomegalovirus, and varicela zoster can act as triggers for PV. This process is not immediate after the infection because it depends on the change in antibodies initially produced against the virus that start to identify antigens present in the skin's anchoring structures. SARS-CoV-2 can induce autoimmunity, as seen in Guillán-Barré syndrome and in Kawasaki disease. It is a highly immunogenic virus that is the perfect agent for triggering PV. This case can be considered a cutaneous autoimmune post-COVID-19 manifestation.
\end{abstract}

Keywords COVID-19 $\cdot$ SARS-CoV-2 $\cdot$ Autoimmunity $\cdot$ Emerging infections $\cdot$ Viruses $\cdot$ Pemphigus

\section{Introduction}

Bullous autoimmune diseases are a group of disorders characterized by blister formation on the skin and/or mucosa due to autoantibodies against cellular anchorage structures of

This article is part of the Topical Collection on Covid-19

Vanessa Lucilia Silveira De Medeiros

vanessalucilia@yahoo.com.br

1 Dermatology Clinics, Tropical Medicine Department, Clinics Hospital, Federal University of Pernambuco, Recife, PE, Brazil

2 Unimed Recife, Unimed Recife III Hospital, Recife, Brazil

3 Medical Faculty of Recife, Medical Sciences Center, Federal University of Pernambuco, Recife, PE, Brazil

4 Multihemo Oncoclínicas, Recife, PE, Brazil

5 Department of Pathology, Federal University of Pernambuco, Recife, PE, Brazil those sites [1]. Viral diseases are potential inducers of autoimmunity reactions [2].

Coronavirus disease 19 (COVID-19) is a multisystemic disease that may present skin lesions [3, 4]. The most described cutaneous manifestations related to severe acute respiratory syndrome coronavirus 2 (SARS-CoV-2) infection are urticarial lesions [5], chilblain-like lesions, vesicular eruptions, maculopapular rash, and livedo [6]. They can occur alone or with the involvement of other organs and may happen before, during, or after systemic symptoms [5, 6]. Despite the progress made in the knowledge about acute symptoms of COVID-19, many questions remain about the consequences of the inflammatory process induced by the virus.

Autoimmune reactions were described in the context of COVID-19 [7]. However, skin autoimmune phenomena related to SARS-CoV-2 have not yet been reported. We report a case of PV present after SARS-CoV-2 infection and discuss the possible relationship between the virus and autoimmunity phenomena. 


\section{Case Report}

A 43-year-old male without comorbidities was diagnosed with mediastinal Hodgkin's lymphoma stage II in January 2019. The patient had no personal or family history of autoimmunity. He had failed with the first line of treatment (abriamycin/bleomycin/vinblastine/dacarbazine) on June 2019 and after with radiotherapy ended in February 2020. Due to the outbreak of the pandemic, he was without any treatment, on an immunotherapy schedule in July of 2020 when presented anosmia and ageusia without other symptoms. RT-PCR was positive for SARS-CoV-2 on 07/30/ 2020, and there were no laboratory changes. The symptoms resolved spontaneously after 10 days; however, due to infection, the treatment of lymphoma was postponed. Forty days later on 09/10/2020, he presented bullae on the chest. He was treated with acyclovir with a hypothesis of herpes zoster outpatient at the hematology clinic. The bullae spread to the back, abdomen, upper limbs, and lower limbs. He was hospitalized on $09 / 28 / 20$, and the dermatologist's evaluation was requested. On physical examination, he presented monomorphic flaccid bullae over edematous plaques and erosions on the chest, abdomen, between the scapulas, arms, and thighs (Fig. 1). There were small ulcers on the oral mucosa. There were no lesions on the genital or eyes. The patient had no fever or other systemic symptoms. There was no other manifestation of autoimmunity. The diagnostic hypotheses were bullous lesions

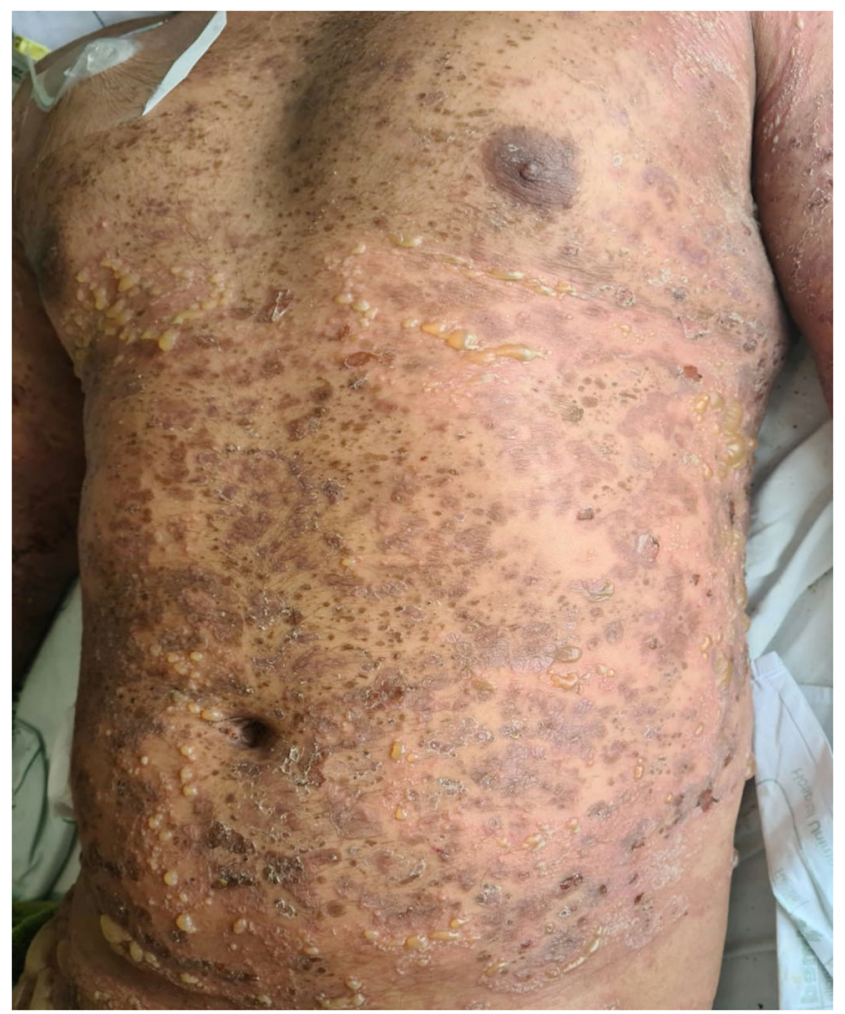

Fig. 1 Large flaccid bullae and erosions on the chest and abdomen due to coronavirus reinfection/reactivation, PV, paraneoplastic pemphigus (PNP), or bullous pemphigoid. The absence of polymorphism and the mild involvement of the mucosa were against PNP. There were no pustular lesions to include IgA PV in the hypotheses. RT-PCR for SARSCoV-2 was repeated, and the result was negative. Laboratory exams were normal, and there were no signs of other infections. Histopathological examination of the skin and mucosa fragments demonstrated suprabasal acantholytic dermatosis with a mixed inflammatory infiltrate with eosinophils (Fig. 2A) compatible with PV. Direct immunofluorescence (DIF) showed intracellular IgG (Fig. 2B) and C3 with negative IgA, confirming PV. The patient was treated with prednisone $1 \mathrm{mg} / \mathrm{kg}$ for 7 days with a good response, and subcutaneous methotrexate (MTX) $15 \mathrm{mg} /$ week was added. The patient was discharged after 15 days on 10/13/19 with oral MTX $15 \mathrm{mg} /$ week and prednisone $60 \mathrm{mg}$ per day. The patient had a good evolution with a reduction of prednisone for $20 \mathrm{mg}$ combined with MTX $15 \mathrm{mg}$ per week.

\section{Discussion}

Autoimmunity development is a complex process and may be idiopathic or induced by neoplasms, drugs, infections, or inflammatory processes [7]. There are different mechanisms that result in autoimmunity, such as molecular mimicry, immune activation by exposure to self-antigens (bystander activation), and subsequent expansion of autoreactive $\mathrm{T}$ cells by the epitope spreading phenomenon in some cases $[2,8]$.

The autoimmunity process starts with massive migration of CD8+ T cells to the site of infection, resulting in target cell death and posterior phagocytosis by macrophages. Eventually, there is a natural similarity between viral and host antigens, leading to autoantigen identification and destruction by the immune system, a mechanism called molecular mimicry. When there is no similarity of antigens but the cytolysis process is greater than phagocytosis, self-antigens can be exposed to the immune system, generating autoreactive cells [2, 8]. This phenomenon is called bystander activation. In the case of prolonged viral disease or the presence of a virus with multiple antigenic targets, the immune system optimizes its response to attack the infectious agent in as many epitopes as possible, amplifying the recognized antigens [2, 8]. However, in susceptible individuals, this natural process induces autoimmunity [8]. After the first stage in which bystander activation or molecular mimicry occurs, immune response amplification leads to the identification of different self-antigens not related to the first antigen and, consequently, the development of autoantibodies, a phenomenon called epitope spreading $[2,8]$.

SARS-CoV-2 can be an environmental trigger for autoimmune reactions, possibly through more than one mechanism 
Fig. 2 A Histopathologic exam, magnitude $\times 400$, hematoxylineosin coloration: blister with suprabasal acantholysis. B Direct immunofluorescence positive for intercellular IgG

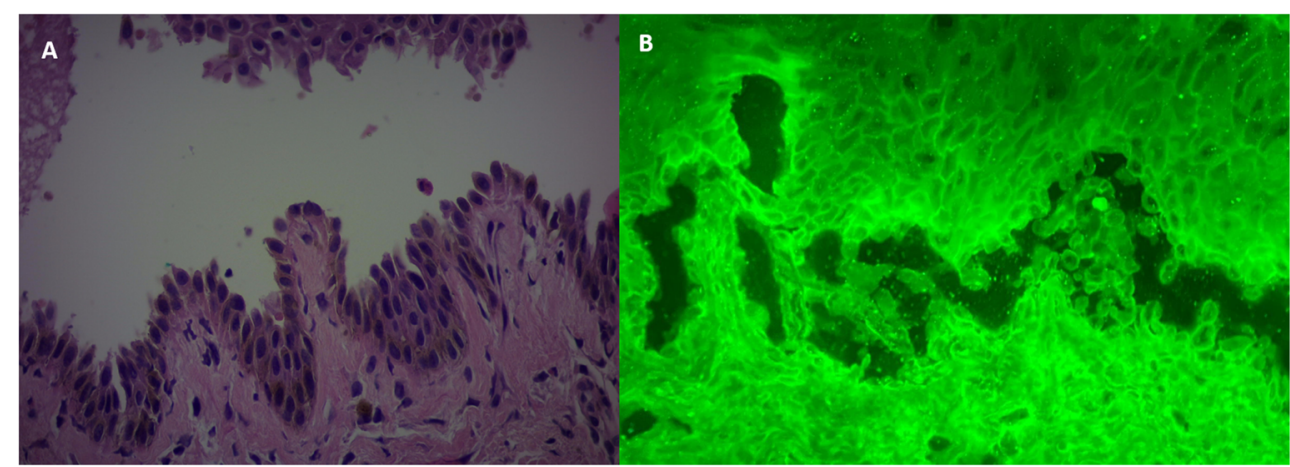

[6]. Guillain-Barré syndrome (GBS), which is most likely presented earlier in the course of the disease, is one of the pathologies triggered by COVID-19 [9-11]. The relationship between GBS and previous viral infections is well established. It probably occurs through molecular mimicry due to a similarity between the virus antigens and the ganglioside components of the peripheral nerves, resulting in the rapid onset of symptoms [6].

A second autoimmune phenomenon due to SARS-CoV-2 infection is Kawasaki disease, reported in children 9 to 45 days after mild COVID-19 [12, 13]. There are many possible etiologies for Kawasaki disease, among which viruses are suggested by a seasonal increase in cases and outbreaks [14] and by the presence of viral RNA in lung biopsies, among which coronavirus [15]. These findings were reported before the pandemic, and until recently, there were those who contested viruses as triggers for Kawasaki disease [14]. With the pandemic, this link has become clearer. Kawasaki is a febrile necrotizing vasculitis caused by infiltration of $\operatorname{IgA}$ plasma cells, CD8+ T lymphocytes, and macrophages in the coronary arteries with an increase in proinflammatory cytokines (IL-1, IL-6, IL-8), a decrease in TNF-alpha, and the presence of autoantibodies $[14,15]$. The autoimmunity mechanism and the epitope responsible for triggering this process are not yet known [15].

There are currently no reports of cutaneous autoimmune lesions due to SARS-CoV-2. However, cutaneous hypersensitivity reactions due to unknown mechanisms were reported during the COVID-19 course [16-18], suggesting that SARSCoV-2 promoted immune dysregulation, inducing a hypersensitive state and skin lesions not directly related to the virus. Pityriasis rosea in children, which occurs 1 week or more after SARS-CoV-2 or herpesvirus (HHV) 7/6 infection, is also a postinfection immunological manifestation $[19,20]$. Although viral fragments can be found in the lesions, they are the result of the migration of $\mathrm{CD}^{+} \mathrm{T}$ lymphocytes and Langerhans cells to the skin in response to systemic infection and may result from the interaction between more than one virus $[19,20]$.

Environmental triggers can induce bullous autoimmune diseases in a genetically susceptible individual due to molecular mimicry or epitope spreading phenomena $[4$, 21]. The herpesvirus family is a high lymphotropic virus and once again is involved in this association. IgG against herpes simplex 1 and cytomegalovirus has been identified in the serum of patients with PV more frequently than patients with pemphigus foliaceus (PF) and healthy controls. DIF for herpesvirus was also positive in the oral mucosa of PV [22]. A case report of PV presented 17 days after a varicella infection again indicates that viruses can act as triggers for the disease [23]. Although we have been in an endemic region for PF [1] since April 2020, near the apex of the first wave of the pandemic, we noticed an increase in PV cases. However, it is difficult to prove a temporal relationship between the facts due to the late appearance of skin symptoms and hospitalizations in hospitals without structure for investigation of dermatological cases [24].

The patient had Hodgkin's lymphoma as a risk factor for PNP and IgA pemphigus; however, the clinical picture and the investigation ruled out these hypotheses $[25,26]$. IgA pemphigus is more associated with $\mathrm{T}$ cell lymphoma. The lesions start with pustules in the trunk or extremities that progress to the arciforme or annular crusted plaques. Biopsy shows intraepidermal or suprabasal neutrophilic infiltrate, and DIF is positive for intercellular IgA in the epidermis [25]. PNP is more common in the course of non-Hodgkin lymphoma and presents clinically with conjunctivitis, intratable mucositis, and polymorphic lesions on the skin due to the variable epitopes targeted by antibodies. The histopathological exam shows different patterns, such as suprabasal acantholysis with scant inflammatory infiltrate, interface dermatitis, or basal cell vacuolar degeneration, reflecting polymorphic lesions. DIF was positive for intercellular IgG and $\mathrm{C} 3$ in $50 \%$ of the cases, but it was distributed more intensively in the cells of the basal layer. Indirect immunofluorescence was positive for IgG in the rat bladder. Immunoblotting or immunoprecipitation shows several proteins, such as desmoplakin, envoplakin, periplakin, bullous pemphigoid antigen 1, and alpha-2microglobulin 1. PNP is poorly responsive to corticosteroids and conventional treatments such as MTX [26]. Clinical picture, histopathologic exam, DIF result, and good response to treatment confirmed PV. 
It could be assumed that lymphoma was responsible for the appearance of PV. However, large population studies did not confirm this association [27]. Lymphoma could help in the autoimmune process, exposing autoantigens and promoting the ideal environment for immunological activation in the context of a highly antigenic viral disease [26]. Autoimmune phenomena can occur after mild covid conditions [9-13]. The late temporal relation between SARS-CoV-2 infection and the emergence of PV was expected since the development of permanent autoimmunity requires a second step after the initial inflammatory process $[8,9]$. PF in its endemic form has some environmental factors in its pathogenesis, however, without knowing when the contact happened.

\section{Conclusions}

SARS-CoV-2 has been reported as a cause of autoimmune manifestations [10-14, 23, 24]. PV was previously associated with viral infections, and the immune mechanism of PV development can be triggered after COVID-19 [21]. These facts suggest that this case can be considered an autoimmune cutaneous post-COVID-19 manifestation.

Author Contribution VLSdM: author of the project, coordination of the research team, clinical evaluation, and text reviewer. AUM-N: clinical evaluation and text writer. DMT: laboratory analysis and text reviewer. RCB: clinical evaluation and text writer. ÉOdMC: clinical evaluation and text writer. DMT: laboratory analysis and text reviewer

Availability of Data and Material All the material is available with the corresponding author.

Code Availability Not applicable

\section{Declarations}

Competing Interests The authors declare no competing interests.

\section{References}

1. Alpsoy E, Akman-Karakas A, Uzun S. Geographic variations in epidemiology of two autoimmune bullous diseases: pemphigus and bullous pemphigoid. Arch Dermatol Res. 2015;307(4):291-8. https://doi.org/10.1007/s00403-014-1531-1.

2. Smatti MK, Cyprian FS, Nasrallah GK, Al Thani AA, Almishal RO, Yassine HM. Viruses and autoimmunity: a review on the potential interaction and molecular mechanisms. Viruses. 2020;11(8): 762. https://doi.org/10.3390/v11080762.

3. Zhou M, Zhang X, Qu J. Coronavirus disease 2019 (COVID-19): a clinical update. Front Med. 2020;14(2):126-35. https://doi.org/10. 1007/s11684-020-0767-8.

4. Spuntarelli V, Luciani M, Bentivegna E, Marini V, Falangone F, Conforti G, et al. COVID-19: is it just a lung disease? A case-based review. SN Compr Clin Med. 2020:1-6. https://doi.org/10.1007/ s42399-020-00418-6.

5. de Medeiros VLS, Silva LFT. Follow-up of skin lesions during the evolution of COVID-19: a case report. Arch Dermatol Res. 2020;14:1-4. https://doi.org/10.1007/s00403-020-02091-0.

6. Galván Casas C, Català A, Carretero Hernández G, RodríguezJiménez P, Fernández-Nieto D, Rodríguez-Villa Lario A, et al. Classification of the cutaneous manifestations of COVID-19: a rapid prospective nationwide consensus study in Spain with 375 cases. Br J Dermatol. 2020;183(1):71-7. https://doi.org/10.1111/bjd. 19163

7. Shah S, Danda D, Kavadichanda C, Das S, Adarsh MB, Negi VS. Autoimmune and rheumatic musculoskeletal diseases as a consequence of SARS-CoV-2 infection and its treatment. Rheumatol Int. 40(10):1539-54. https://doi.org/10.1007/s00296-020-04639-9.

8. Vojdani A. A potential link between environmental triggers and autoimmunity. Autoimmune Dis. 2014;2014:437231-18. https:// doi.org/10.1155/2014/437231.

9. Powell AM, Black MM. Epitope spreading: protection from pathogens, but propagation of autoimmunity? Clin Exp Dermatol. 2001;26(5):427-33. https://doi.org/10.1046/j.1365-2230.2001. 00852.x.

10. Toscano G, Palmerini F, Ravaglia S, Ruiz L, Invernizzi P, Cuzzoni $\mathrm{MG}$, et al. Guillain-Barré syndrome associated with SARS-CoV-2. N Engl J Med. 2020;382(26):2574-6. https://doi.org/10.1056/ NEJMc2009191.

11. Bigaut K, Mallaret M, Baloglu S, Nemoz B, Morand P, Baicry F, et al. Guillain-Barré syndrome related to SARS-CoV-2 infection. Neurol Neuroimmunol Neuroinflamm. 2020;7(5):e785. https://doi. org/10.1212/NXI.0000000000000785.

12. Scheidl E, Canseco DD, Hadji-Naumov A, Bereznai B. GuillainBarré syndrome during SARS-CoV-2 pandemic: a case report and review of recent literature. J Peripher Nerv Syst. 2020;25(2):204-7. https://doi.org/10.1111/jns.12382.

13. Toubiana J, Poirault C, Corsia A, Bajolle F, Fourgeaud J, Angoulvant F, et al. Kawasaki-like multisystem inflammatory syndrome in children during the covid-19 pandemic in Paris, France: prospective observational study. BMJ. 2020;369:m2094. https:// doi.org/10.1136/bmj.m2094.

14. Verdoni L, Mazza A, Gervasoni A, Martelli L, Ruggeri M, Ciuffreda M, et al. An outbreak of severe Kawasaki-like disease at the Italian epicenter of the SARS-CoV-2 epidemic: an observational cohort study. Lancet. 2020;395(10239):1771-8. https://doi. org/10.1016/S0140-6736(20)31103-X.

15. Agarwal S, Agrawal DK. Kawasaki disease: etiopathogenesis and novel treatment strategies. Expert Rev Clin Immunol. 2017;13(3): 247-58. https://doi.org/10.1080/1744666X.2017.1232165.

16. Noval Rivas M, Arditi M. Kawasaki disease: pathophysiology and insights from mouse models. Nat Rev Rheumatol. 2020;16(7):391405. https://doi.org/10.1038/s41584-020-0426-0.

17. Mahé A, Birckel E, Krieger S, Merklen C, Bottlaender L. A distinctive skin rash associated with coronavirus disease 2019? J Eur Acad Dermatol Venereol. 2020;34(6):e246-7. https://doi.org/10. $1111 /$ jdv.16471.

18. Sanchez A, Sohier P, Benghanem S, L'Honneur AS, Rozenberg F, Dupin N, et al. Digitate papulosquamous eruption associated with severe acute respiratory syndrome coronavirus 2 infection. JAMA Dermatol. 2020;156(7):819-20. https://doi.org/10.1001/ jamadermatol.2020.1704.

19. Mahajan K, Relhan V, Relhan AK, Garg VK. Pityriasis rosea: an update on etiopathogenesis and management of difficult aspects. Indian J Dermatol. 2016;61(4):375-84. https://doi.org/10.4103/ 0019-5154.185699.

20. Drago F, Broccolo F, Rebora A. Pityriasis rosea: an update with a critical appraisal of its possible herpesviral etiology. J Am Acad 
Dermatol. 2009;61(2):303-18. https://doi.org/10.1016/j.jaad.2008. 07.045 .

21. Brunasso AMG, Castellaneta M, Pontali E, Raggi F, Massone C. Follow-up of skin lesions during COVID-19: a description of a DRESS case. Arch Dermatol Res. 2021;6:1-4. https://doi.org/10. 1007/s00403-020-02171-1.

22. Goon AT, Tay YK, Tan SH. Pemphigus vulgaris following varicella infection. Clin Exp Dermatol. 2001;26(8):661-3. https://doi. org/10.1046/j.1365-2230.2001.00912.

23. Didona D, Di Zenzo G. Humoral epitope spreading in autoimmune bullous diseases. Front Immunol. 2018;9:779. https://doi.org/10. 3389/fimmu.2018.00779.

24. Machado ARDSR, La Serra L, Turatti A, Machado AM, Roselino AM. Herpes simplex virus 1 and cytomegalovirus are associated with pemphigus vulgaris but not with pemphigus foliaceus disease. Exp Dermatol. 2017;26(10):966-8. https://doi.org/10.1111/exd. 13342.
25. Maruta CW, Miyamoto D, Aoki V, Carvalho RGR, Cunha BM, Santi CG. Paraneoplastic pemphigus: a clinical, laboratorial, and therapeutic overview. An Bras Dermatol. 2019;94(4):388-98. https://doi.org/10.1590/abd1806-4841.20199165.

26. Chen L, Yang B, Fan J, Yang K, Liu H, Wu G. Peripheral T-cell lymphoma complicated by immunoglobulin A pemphigus: a case report and literature review. Oncol Lett. 2014;8(1):62-6. https://doi. org/10.3892/ol.2014.2088.

27. Kridin K, Zelber-Sagi S, Comaneshter D, Cohen AD. Coexistent solid malignancies in pemphigus: a population-based study. JAMA Dermatol. 2018;154(4):435-40. https://doi.org/10.1001/ jamadermatol.2017.6334.

Publisher's Note Springer Nature remains neutral with regard to jurisdictional claims in published maps and institutional affiliations. 